\title{
Coincidence of two Swan conductors of abelian characters
}

\author{
Kazuya Kato and Takeshi Saito
}

\begin{abstract}
There are two ways to define the Swan conductor of an abelian character of the absolute Galois group of a complete discrete valuation field. We prove that these two Swan conductors coincide.
\end{abstract}

Keywords. Local field, ramification groups, Swan conductor

2010 Mathematics Subject Classification. 11S15; 14B25

[Français]

Titre. Coïncidence de deux conducteurs de Swan des caractères abéliens

Résumé. Il y a deux façons de définir le conducteur de Swan d'un caractère abélien du groupe de Galois absolu d'un corps de valuation discrète complet. Nous montrons que ces deux conducteurs de Swan coïncident.

Received by the Editors on April 24, 2019, and in final form on August 19, 2019. Accepted on September 23, 2019.

Kazuya Kato

Department of Mathematics, University of Chicago, Chicago, IL, USA

e-mail: kkato@math.uchicago.edu

Takeshi Saito

School of Mathematical Sciences, University of Tokyo, Tokyo 153-8914, Japan

e-mail: t-saito@ms.u-tokyo.ac.jp 


\section{Contents}

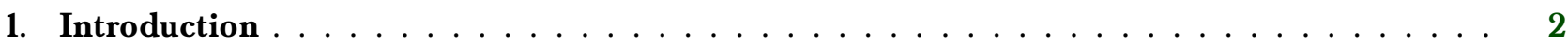

2. On the theorem of Epp $\ldots \ldots \ldots \ldots \ldots \ldots \ldots$

3. Some extensions of complete discrete valuation fields $\ldots \ldots \ldots \ldots$

4. Review and complements on ramification groups $\ldots \ldots \ldots \ldots$

5. Coincidence of Swan conductors and of refined Swan conductors $\ldots \ldots \ldots$

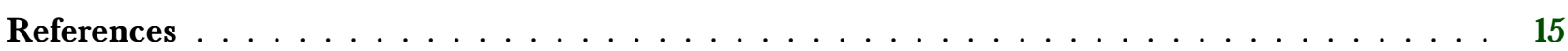

\section{Introduction}

1.1. Let $K$ be a complete discrete valuation field, let $\bar{K}$ be a separable closure of $K$, and let us consider $\chi: \operatorname{Gal}(\bar{K} / K) \rightarrow \mathbb{C}^{\times}$a homomorphism which factors through $\mathrm{Gal}(L / K)$ for a finite cyclic extension $L \subset \bar{K}$ of $K$. There are two definitions of the Swan conductor of $\chi$, one is defined by using the logarithmic upper ramification filtration on $\operatorname{Gal}(\bar{K} / K)$ defined geometrically [1], and the other is defined by using the filtrations on the unit groups of complete discrete valuation fields and cup products in Galois cohomology [6].

We prove that the two Swan conductors coincide.

1.2. We briefly review the two Swan conductors, which we denote in this paper by $\operatorname{Sw}(\chi)$ and $\operatorname{Sw}^{\mathrm{ab}}(\chi)$, respectively.

$\mathrm{Sw}(\chi)$ is defined as follows. There is a decreasing filtration $\operatorname{Gal}(\bar{K} / K)_{\log }^{t}$ indexed by $t \in \mathbb{Q}_{\geq 0}$ on $\operatorname{Gal}(\bar{K} / K)$ by closed normal subgroups called the logarithmic upper ramification groups. $\mathrm{Sw}(\chi)$ is defined to be the smallest $t \in \mathbb{Q}_{\geq 0}$ such that $\chi\left(\operatorname{Gal}(\bar{K} / K)_{\log }^{s}\right)=\{1\}$ for all $s>t$ (such $t$ exists). See [1, Theorem 3.16] and Section 4.

$\mathrm{Sw}^{\mathrm{ab}}(\chi)$ is defined as follows. Fix an injection $\mathbb{Q} / \mathbb{Z} \rightarrow \mathbb{C}^{\times}$, say $r \mapsto \exp (2 \pi \sqrt{-1} r)$, and identify $\chi$ with an element of $H^{1}(K, \mathbb{Q} / \mathbb{Z})=H^{2}(K, \mathbb{Z})$. Then the cup-product with $\chi$ defines a homomorphism $K^{\times} \rightarrow \operatorname{Br}(K)$, denoted by $a \mapsto\{\chi, a\}$, where $\operatorname{Br}(K)=H^{2}\left(K, \mathbb{G}_{m}\right)$ is the Brauer group of $K[13$, Chapitre X.4]. $\mathrm{Sw}^{\mathrm{ab}}(\chi)$ is defined to be the smallest integer $n \geq 0$ such that $\left\{\chi, 1+\mathfrak{m}_{K}^{n} \mathfrak{m}_{K^{\prime}}\right\}=0$ in $\operatorname{Br}\left(K^{\prime}\right)$ for any extension $K \rightarrow K^{\prime}$ of complete discrete valuation fields (such $n$ exists; here $\mathfrak{m}_{*}$ denote the maximal ideals). See [7].

Theorem 1.3. $\mathrm{Sw}(\chi)=\mathrm{Sw}^{\mathrm{ab}}(\chi)$.

1.4. We will also prove the coincidence of refined Swan conductors. Let $r=\operatorname{Sw}(\chi)=\operatorname{Sw}^{\mathrm{ab}}(\chi)$ and assume $r>0$. Then we have non-zero elements

$$
\begin{gathered}
\operatorname{rsw}(\chi) \in \bar{F} \otimes_{F} \mathfrak{m}_{K}^{-r} / \mathfrak{m}_{K}^{-r+1} \otimes_{\mathcal{O}_{K}} \Omega_{\mathcal{O}_{K}}^{1}(\log ), \\
\operatorname{rsw}^{\mathrm{ab}}(\chi) \in \mathfrak{m}_{K}^{-r} / \mathfrak{m}_{K}^{-r+1} \otimes_{\mathcal{O}_{K}} \Omega_{\mathcal{O}_{K}}^{1}(\log )
\end{gathered}
$$

called the (logarithmic) refined Swan conductors. $\operatorname{rsw}(\chi)$ is defined in [10] as a refined version of $\operatorname{Sw}(\chi)$ and $\mathrm{rsw}^{\mathrm{ab}}(\chi)$ is defined in [7] as a refined version of $\mathrm{Sw}^{\mathrm{ab}}(\chi)$. The definition of $\mathrm{rsw}(\chi)$ is recalled in (4.16).

Theorem 1.5. $\operatorname{rsw}(\chi)=\operatorname{rsw}^{\mathrm{ab}}(\chi)$. 
1.6. Theorem 1.3 was known, for example, in the following cases. (The cases (i) and (ii) follow from Section 6.1 of [1] and from [6]. The case (iii) is shown in [2, Corollary 9.12].) The case (ii) is proved by comparing Propositions 4.11 and 5.2. In the cases (i) and (ii), the ring $\mathcal{O}_{L}$ is generated by a single element over $\mathcal{O}_{K}$ and such an extension is also studied in [14].

(i) (The classical case.) The case where the residue field of $K$ is perfect.

(ii) The case where the residue field $F$ of $K$ is of characteristic $p$ such that $\left[F: F^{p}\right]=p$ and $\chi$ factors through $\mathrm{Gal}(L / K)$ for a finite cyclic extension $L / K$ whose ramification index $e(L / K)$ is one.

(iii) The case where $K$ is of positive characteristic.

In the positive characteristic case, the results corresponding to Theorems 1.3 and 1.5 in the non-logarithmic case are proved in [2] and [15].

1.7. Our method to prove Theorem 1.3 is to reduce it to the above case (ii) (not to the classical case (i)). In Theorem 3.1, we prove that for a finite cyclic extension $L / K$ such that $\chi$ factors through $G a l(L / K)$, we can find an extension of complete discrete valuation fields $K \rightarrow K^{\prime}$ such that $\operatorname{Sw}\left(\chi_{K^{\prime}}\right)=e\left(K^{\prime} / K\right) \operatorname{Sw}(\chi)$, $\mathrm{Sw}^{\mathrm{ab}}\left(\chi_{K^{\prime}}\right)=e\left(K^{\prime} / K\right) \mathrm{Sw}^{\mathrm{ab}}(\chi)$, the residue field $F^{\prime}$ of $K^{\prime}$ satisfies $\left[F^{\prime}:\left(F^{\prime}\right)^{p}\right]=p$, and $e\left(L K^{\prime} / K^{\prime}\right)=1$. The refined Swan conductors play important roles to find the field $K^{\prime}$ above.

Theorem 1.5 is proved also by the reduction to the case (ii). The authors would like to thank an anonymous referee for pointing out that almost the same result as the key step Proposition 4.11 is proved in [5, Theorem 5.9].

One of the authors (K. K.) is partially supported by NSF Award 1601861 and (T. S.) is partially supported by JSPS Grant-in-Aid for Scientific Research (A) 26247002.

\section{On the theorem of Epp}

The following theorem is not explicitly written in the paper [4] of Epp, but the arguments there (with a correction in [8] of an error in [4]) actually prove this.

Theorem 2.1. Let $K$ be a complete discrete valuation field whose residue field $F$ is of characteristic $p>0$, and let $L$ be a finite Galois extension of $K$. Then there exists a finite extension $K^{\prime}$ of $K$ satisfying the following conditions (i) and (ii).

(i) $e\left(L K^{\prime} / K^{\prime}\right)=1$.

(ii) The residue field of $K^{\prime}$ is a separable extension of that of $K$.

In Theorem 2.1, we may take $K^{\prime}$ separable over $K$, although we will not use this fact. To see this, it suffices to modify the construction of $K^{\prime}=K\left(\pi^{\prime}\right)$ in the proof of the case where $K$ is of characteristic $p>0$ and $T$ is not empty in 2.6.

We use the following lemmas 2.2, 2.3 and 2.4 for the proof of Theorem 2.1.

For a discrete valuation field $K$, let $\operatorname{ord}_{K}$ be the normalized additive valuation of $K$. In the case the residue field of $K$ is of characteristic $p>0$, let $e_{K}=\operatorname{ord}_{K}(p)$. (So, $e_{K}=\infty$ if $K$ is of characteristic $p$.)

Lemma 2.2. Let $K$ be a complete discrete valuation field whose residue field $F$ is of characteristic $p>0$. Let $k=\bigcap_{r \geq 0} F^{p^{r}}$ be the largest perfect subfield of $F$ and let $W(k) \rightarrow \mathcal{O}_{K}$ be the canonical morphism from the ring of Witt vectors. Then the subring $\bigcap_{r \geq 0}\left(\mathcal{O}_{K} / p \mathcal{O}_{K}\right)^{p^{r}} \subset \mathcal{O}_{K} / p \mathcal{O}_{K}$ equals the image of $k \rightarrow \mathcal{O}_{K} / p \mathcal{O}_{K}$ (in the case $K$ is of characteristic $p$, this means that $\left.\bigcap_{r \geq 0}\left(\mathcal{O}_{K}\right)^{p^{r}}=k\right)$.

Proof. Let $A=\bigcap_{r \geq 0}\left(\mathcal{O}_{K} / p \mathcal{O}_{K}\right)^{p^{r}}$ denote the subring. Then, $A \subset \mathcal{O}_{K} / p \mathcal{O}_{K}$ contains the image of $k$ and the image of $A$ by $\mathcal{O}_{K} / p \mathcal{O}_{K} \rightarrow F$ is a subring of $k$. Hence, the assertion follows from $A \cap\left(\mathfrak{m}_{K} / p \mathcal{O}_{K}\right)=0$. 
We do not give proofs of the following lemmas 2.3 and 2.4 which are straightforward.

Lemma 2.3. Let $K$ be a complete discrete valuation field of characteristic $p>0$ and let $F$ be its residue field. Consider the Artin-Schreier extension $L=K(\alpha), \alpha^{p}-\alpha=f \in K$. Let $\pi$ be a prime element of $K$. Let $E$ be the residue field of $L$.

(1) If $f \in \mathcal{O}_{K}$, the extension $L / K$ is unramified, possibly trivial.

(2) Assume that $-\operatorname{ord}_{K} f=n \geq 1$ is not divisible by $p$. Then $e(L / K)=p$ and $E=F$.

(3) Assume that $f \in u \pi^{-m p}+\pi^{-m p+1} \mathcal{O}_{K}$ for some integer $m \geq 1$ and for some $u \in \mathcal{O}_{K}$ whose residue class $\bar{u}$ does not belong to $F^{p}$. Then $E=F\left(\bar{u}^{1 / p}\right)$ and $e(L / K)=1$.

Lemma 2.4. Let $K$ be a complete discrete valuation field of mixed characteristic $(0, p)$. Let $F$ be its residue field. Assume that $K$ contains a primitive $p$-th root $\zeta_{p}$ of 1 . Consider the Kummer extension $L=K(\alpha), \alpha^{p}=a \in K^{\times}$. Let $\pi$ be a prime element of $K$. Let $E$ be the residue field of $L$.

(1) If $a \in 1+\left(\zeta_{p}-1\right)^{p} \mathcal{O}_{K}$, the extension $L / K$ is unramified, possibly trivial.

(2) Assume that $\operatorname{ord}_{K}(a)$ is not divisible by $p$. Then $e(L / K)=p$ and $E=F$.

(3) Assume that $a \in\left(\mathcal{O}_{K}\right)^{\times}$and that the residue class $\bar{a}$ of a is not contained in $F^{p}$. Then $E=F\left(\bar{a}^{1 / p}\right)$ and $e(L / K)=1$.

(4) Assume that $a \in\left(1+\pi^{n} u\right)\left(1+\pi^{n+1} \mathcal{O}_{K}\right)$ for some integer $n$ not divisible by $p$ such that $1 \leq n<e_{K} p /(p-1)$ and for some $u \in\left(\mathcal{O}_{K}\right)^{\times}$. Then $e(L / K)=p$ and $E=F$.

(5) Assume that $a \in\left(1+\pi^{m p} u\right)\left(1+\pi^{n+1} \mathcal{O}_{K}\right)$ for some integer $m$ such that $1 \leq n=m p<e_{K} p /(p-1)$ and for some $u \in \mathcal{O}_{K}$ whose residue class $\bar{u}$ does not belong to $F^{p}$. Then $E=F\left(\bar{u}^{1 / p}\right)$ and $e(L / K)=1$.

2.5. We start the proof of Theorem 2.1.

First, we reduce the theorem to the case $\left(^{*}\right)$ below. Let $K_{1} \subset L$ be the maximum tamely ramified extension of $K$. Then, since $L K_{1}=L$ and the residue field of $K_{1}$ is a separable extension of that of $K$, we may assume that $\mathrm{Gal}(L / K)$ equals the inertia subgroup $I$ and is a $p$-group.

We prove the reduction by induction on the order of $I$. We may assume that $L \neq K$. Then, since $\operatorname{Gal}(L / K)$ is nilpotent, there exists a subextension $L^{\prime} \subset L$ such that $L^{\prime}$ is a Galois extension over $K$ and that $L$ is a cyclic extension of $L^{\prime}$ of degree $p$. By induction hypothesis, there exists a finite extension $K_{1}^{\prime}$ of $K$ such that $e\left(L^{\prime} K_{1}^{\prime} / K_{1}^{\prime}\right)=1$ and satisfying (ii). If $e\left(L K_{1}^{\prime} / L^{\prime} K_{1}^{\prime}\right)=1$, there is nothing to prove. Otherwise, for the maximum unramified extension $K_{2}^{\prime}$ of $K_{1}^{\prime}$ inside $M_{1}^{\prime}=L^{\prime} K_{1}^{\prime}$, the extensions $K_{2}^{\prime} \subset M_{1}^{\prime} \subset L K_{1}^{\prime}$ satisfies the condition $(*)$.

$\left(^{*}\right)$ There exists a field $M$ such that $K \subset M \subset L, e(M / K)=1$ and that $L$ is a cyclic extension of $M$ of degree $p$ and $e(L / M)=p$. The residue field $E$ of $M$ is a purely inseparable extension of the residue field $F$ of $K$.

2.6. We prove Theorem 2.1 in the case $K$ is of characteristic $p$. Let $M$ be as in $\left(^{*}\right)$ in 2.5 . We may assume $M=E((\pi))$ with $\pi$ a prime element of $K$. We can write $L=M(\alpha)$ where $\alpha^{p}-\alpha=f=f_{I}+f_{U}$ with $f_{I}, f_{U} \in M$ such that: $f_{I}=\sum_{n \in I} a_{n} \pi^{-n}$ where $I$ is a finite subset of $\mathbb{Z}_{>0}$ and $a_{n} \in E^{\times}$, and $f_{U} \in \mathcal{O}_{M}$. By Lemma 2.3 (1) applied to the extension $L / M, I$ is not empty because $L / M$ is not unramified. .

In the following, we use the fact that for $u, v \in M$ such that $u \equiv v \bmod \left\{w^{p}-w \mid w \in M\right\}$, the extension $M(\beta), \beta^{p}-\beta=u$, of $M$ is the same as that given by $v$. If $n \in I$ is divisible by $p$ and $a_{n} \in E^{p}$, we have $a_{n} \pi^{-n} \equiv a_{n}^{1 / p} \pi^{-n / p} \bmod \left\{w^{p}-w \mid w \in M\right\}$ and hence we can replace $a_{n} \pi^{-n}$ by $a_{n}^{1 / p} \pi^{-n / p}$. Hence we may (and do) assume that if $n \in I$ is divisible by $p$, then $a_{n} \notin E^{p}$. 
Let $S$ be the subset of $I$ consisting of all $n \in I$ such that $a_{n} \in \bigcap_{r \geq 0} E^{p^{r}}=\bigcap_{r \geq 0} F^{p^{r}}=k$, and let $T=I \backslash S$. Note that if $n \in S$, then $n$ is not divisible by $p$. By Lemma 2.2, we have $a_{n} \in k \subset \mathcal{O}_{K}$ for $n \in S$. Hence $f_{S} \in K$.

Assume first $T$ is empty. Then $f_{I}=f_{S} \in K$. For $K^{\prime}=K\left(\alpha_{S}\right)$ with $\alpha_{S}^{p}-\alpha_{S}=f_{S}$, the residue field of $K^{\prime}$ coincides with $F$ by Lemma 2.3 (2) applied to $K^{\prime} / K$, and the extension $L K^{\prime} / M K^{\prime}$ is unramified by Lemma 2.3 (1) applied to $L K^{\prime} / M K^{\prime}$.

Assume that $T$ is not empty. For $n \in T$, write $a_{n}=b_{n}^{p^{r(n)}}$ where $b_{n} \in E, r(n) \geq 0$, and $b_{n}$ is not a $p$-th power in $E$. Take an integer $m$ such that $m>r(n)$ for any $n \in T$. For $n \in S$, write $a_{n}=b_{n}^{p^{m}}\left(b_{n} \in k \subset \mathcal{O}_{K}\right)$.

Let $K^{\prime}=K\left(\pi^{\prime}\right)$ where $\pi^{\prime}$ is a $p^{m}$-th root of $\pi$ and let $M^{\prime}=M K^{\prime}, L^{\prime}=L K^{\prime}$. Then

$$
\begin{gathered}
f_{I} \equiv f_{S}+f_{T} \bmod \left\{w^{p}-w \mid w \in M^{\prime}\right\} \\
f_{S}=\sum_{n \in S} b_{n}\left(\pi^{\prime}\right)^{-n}, \quad f_{T}=\sum_{n \in T} b_{n}\left(\pi^{\prime}\right)^{-n p^{m-r(n)}} .
\end{gathered}
$$

Note that $f_{S} \in k\left(\left(\pi^{\prime}\right)\right) \subset K^{\prime}$ by Lemma 2.2. Let $n_{S}:=\max (S)$ and $n_{T}:=\max \left\{n p^{m-r(n)} \mid n \in T\right\}$. If $S$ is empty, we set $n_{S}=1$ so that we have $n_{S}<n_{T}$. Since $n_{S}$ is not divisible by $p$ and $n_{T}$ is divisible by $p$, we have $n_{S} \neq n_{T}$. For the proof of Theorem 2.1, it is sufficient to prove the following Claim 1 and Claim 2.

Claim 1. If $n_{S}<n_{T}$, then $e\left(L^{\prime} / M^{\prime}\right)=1$.

Claim 2. If $n_{S}>n_{T}$, let $K^{\prime \prime}=K^{\prime}(\beta)$ where $\beta^{p}-\beta=f_{S}$ and let $M^{\prime \prime}=M K^{\prime \prime}, L^{\prime \prime}=L K^{\prime \prime}$. Then the residue field of $K^{\prime \prime}$ coincides with that of $K$ and $e\left(L^{\prime \prime} / M^{\prime \prime}\right)=1$.

We first prove

Claim 3. There is a unique $n \in T$ such that $n p^{m-r(n)}=n_{T}$.

We prove Claim 3. If $n, n^{\prime} \in T, n>n^{\prime}$ and $n p^{m-r(n)}=n^{\prime} p^{m-r\left(n^{\prime}\right)}$, then by $n=n^{\prime} p^{r(n)-r\left(n^{\prime}\right)}>n^{\prime}$, we have $p \mid n$. Hence $a_{n} \notin E^{p}$ and $r(n)=0$. This contradicts to $r(n)>r\left(n^{\prime}\right)$.

Claim 1 follows from Claim 3 and Lemma 2.3 (3) applied to the extension $L^{\prime} / M^{\prime}$.

We prove Claim 2. We have $e\left(K^{\prime \prime} / K^{\prime}\right)=p$ by Lemma $2.3(2)$ applied to $K^{\prime \prime} / K^{\prime}$. If $\tau$ denotes a prime element of $K^{\prime \prime}$, the residue class of the unit $\tau^{p}\left(\pi^{\prime}\right)^{-1}$ is a $p$-th power. Claim 2 follows from this and Claim 3 , and from Lemma 2.3 (3) applied to the extension $L^{\prime \prime} / M^{\prime \prime}$.

2.7. We prove Theorem 2.1 in the case $K$ is of mixed characteristic $(0, p)$. We may assume that $K$ contains a primitive $p$-th root $\zeta_{p}$ of 1 . Note that $\operatorname{ord}_{K}\left(\zeta_{p}-1\right)=e_{K} /(p-1)$. Let $M$ be as in $\left(^{*}\right)$ in 2.5. We have $L=M(\alpha), \alpha^{p}=a$ for $a \in M^{\times}$.

The proof consists of two steps. In Step 1, we show that we may assume $a \in 1+p \mathcal{O}_{M}$. In Step 2, we give the proof assuming $a \in 1+p \mathcal{O}_{M}$.

Let $E$ be the residue field of $M$ and take a ring homomorphism $E \rightarrow \mathcal{O}_{M} / p \mathcal{O}_{M}$ such that the induced map $E \rightarrow \mathcal{O}_{M} / \mathfrak{m}_{M}=E$ is the identity map, and its lifting $\iota: E \rightarrow \mathcal{O}_{M}$. Let $\pi$ be a prime element of $K$.

Step 1. Write $a \equiv c \prod_{n \in T} c_{n} \bmod 1+p \mathcal{O}_{M}$ where $T$ is a subset of $\left\{0, \ldots, e_{M}-1\right\}$ and $c_{n}(n \in T)$ and $c$ are elements of $M^{\times}$of the following form. If $0 \in T, c_{0}=\iota(b)$ for some $b \in E$ such that $b \notin E^{p}$. If $n \in T$ and $n \geq 1, c_{n}=1+\pi^{n} \iota(b)$ for some $b \in E$ such that $b \notin E^{p}$. The first term $c$ is a product of a power of $\pi$ and elements of the form $1+\pi^{m} l(b)$ with $b \in E^{p}$ for some integer $m \geqq 0$.

Let $K^{\prime}=K\left(\pi^{1 / p}\right), M^{\prime}=M K^{\prime}, L^{\prime}=L K^{\prime}$. Then we have $c \in\left(\left(M^{\prime}\right)^{\times}\right)^{p}\left(1+p \mathcal{O}_{M^{\prime}}\right)$ since the map $x \mapsto x^{p}$ on $\mathcal{O}_{M^{\prime}} / p \mathcal{O}_{M^{\prime}}$ is a ring homomorphism. Hence if $T \neq \varnothing$, we have $e\left(L^{\prime} / M^{\prime}\right)=1$ by Lemma 2.4 (3) and (5) applied to the extension $L^{\prime} / M^{\prime}$. If $T=\varnothing$, we also have $L^{\prime}=M^{\prime}(\beta)$ with $\beta^{p} \in 1+p \mathcal{O}_{M^{\prime}}$. Thus, the assertion is reduced to the case where $L=M(\alpha), \alpha^{p}=a$ for $a \in 1+p \mathcal{O}_{M}$.

Step 2. Assume $L=M(\alpha), \alpha^{p}=a \in 1+p \mathcal{O}_{M}$. We have an isomorphism

$$
\left(\zeta_{p}-1\right)^{-1} \mathcal{O}_{M} / \mathcal{O}_{M} \rightarrow\left(1+p \mathcal{O}_{M}\right) /\left(\left(1+\left(\zeta_{p}-1\right)^{p} \mathcal{O}_{M}\right) ; x \mapsto 1+\left(\zeta_{p}-1\right)^{p} x\right.
$$


(from the additive group to the multiplicative group). This isomorphism maps $x^{p}-x$ for $x \in\left(\zeta_{p}-1\right)^{-1} \mathcal{O}_{M}$ such that $x^{p} \in\left(\zeta_{p}-1\right)^{-1} \mathcal{O}_{M}$ to a $p$-th power because

$$
\left(1+\left(\zeta_{p}-1\right) x\right)^{p} \equiv 1+\left(\zeta_{p}-1\right)^{p}\left(x^{p}-x\right) \bmod 1+\left(\zeta_{p}-1\right)^{p} \mathcal{O}_{M}
$$

Hence we have a situation similar to the theory of Artin-Schreier extension, and the rest of the proof, which is given below, is similar to the proof of the case where $K$ is of characteristic $p$ in 2.6.

We have $a=a_{I} a_{U}$ with $a_{I}=1+\sum_{n \in I}\left(\left(\zeta_{p}-1\right)^{p} \pi^{-n}\right) \iota\left(a_{n}\right)$ where $I$ is a subset of $\left\{n \in \mathbb{Z} \mid 1 \leq n \leq e_{M} /(p-1)\right\}$ and $a_{n} \in E^{\times}$and $a_{U} \in 1+\left(\zeta_{p}-1\right)^{p} \mathcal{O}_{M}$. Note that we have $\left(\zeta_{p}-1\right)^{p} \pi^{-n} \in p \mathcal{O}_{M}$ for $n \in I$. By Lemma 2.4 (1) applied to the extension $L / M, I$ is not empty. We may assume that if $n \in I$ and $n$ is divisible by $p$, then $a_{n}$ is not a $p$-th power in $E$. Let $k=\bigcap_{r \geq 0} F^{p^{r}}=\bigcap_{r \geq 0} E^{p^{r}}, S=\left\{n \in I \mid a_{n} \in k\right\}$ and let $T=I \backslash S$.

If $T$ is empty, by Lemma 2.2, we have $a_{I} \equiv c \bmod \left(M^{\times}\right)^{p}$ where $c=1+\sum_{n \in S}\left(\left(\zeta_{p}-1\right)^{p} \pi^{-n}\right)\left[a_{n}\right]$ for the Teichmüller lifting $\left[a_{n}\right] \in W(k)^{\times} \subset \mathcal{O}_{K}^{\times}$. Let $K^{\prime}=K\left(c^{1 / p}\right)$. Then the residue field of $K^{\prime}$ is $F$ by Lemma 2.4 (4) applied to the extension $K^{\prime} / K$ and the residue field of $K^{\prime}$ is the same as that of $K$, and the extension $L K^{\prime} / M K^{\prime}$ is unramified by Lemma 2.4 (1) applied to $L K^{\prime} / M K^{\prime}$.

Assume now that $T$ is not empty. For $n \in T$, define $b_{n} \in E \backslash E^{p}$ and $r(n) \geq 0$ as in 2.6. Further take an integer $m$ such that $m>r(n)$ for any $n \in T$ and $b_{n} \in k$ for $n \in S$ as in 2.6.

Let $K^{\prime}=K\left(\pi^{\prime}\right)$ where $\pi^{\prime}$ is a $p^{m}$-th root of $\pi$ and let $M^{\prime}=M K^{\prime}, L^{\prime}=L K^{\prime}$ Then by Lemma 2.2,

$$
\begin{gathered}
a_{I} \equiv a_{S} a_{T} \bmod \left(1+\left(\zeta_{p}-1\right)^{p} \mathcal{O}_{M^{\prime}}\right), \\
K^{\prime} \ni a_{S}=1+\sum_{n \in S}\left(\left(\zeta_{p}-1\right)^{p}\left(\pi^{\prime}\right)^{-n}\right)\left[b_{n}\right], \quad a_{T}=1+\sum_{n \in T}\left(\left(\zeta_{p}-1\right)^{p}\left(\pi^{\prime}\right)^{-n p^{m-r(n)}}\right) \iota\left(b_{n}\right)
\end{gathered}
$$

where $\left[b_{n}\right] \in W(k)^{\times} \subset \mathcal{O}_{K}^{\times}$for $n \in S$ is the Teichmüller lifting of $b_{n}$. Let $n_{T}:=\max \left\{n p^{m-r(n)} \mid n \in T\right\}$ and $n_{S}:=\max (S)$. Since $n_{S}$ is not divisible by $p$ and $n_{T}$ is divisible by $p$, we have $n_{S} \neq n_{T}$. For the proof of Theorem 2.1, it is sufficient to prove the following Claim 1 and Claim 2.

Claim 1. If $n_{S}<n_{T}$, then $e\left(L^{\prime} / M^{\prime}\right)=1$.

Claim 2. If $n_{S}>n_{T}$, let $K^{\prime \prime}=K^{\prime}\left(a_{S}^{1 / p}\right)$. Then the residue field of $K^{\prime \prime}$ coincides with that of $K$ and $e\left(L^{\prime \prime} / M^{\prime \prime}\right)=1$ where $L^{\prime \prime}=L K^{\prime \prime}$ and $M^{\prime \prime}=M K^{\prime \prime}$.

We first prove

Claim 3. There is a unique $n \in T$ such that $n p^{m-r(n)}=n_{T}$.

The proof of Claim 3 is similar to that of Claim 3 in 2.6. Claim 1 follows from Claim 3 and Lemma 2.4 (5) applied to the extension $L^{\prime} / M^{\prime}$. We prove Claim 2. The residue field of $K^{\prime \prime}$ is $F$ by Lemma $2.4(4)$ applied to the extension $K^{\prime \prime} / K^{\prime}$ and we have $e\left(K^{\prime \prime} / K^{\prime}\right)=p$. If $\tau$ denotes a prime element of $K^{\prime \prime}$, the residue class of the unit $\tau^{p}\left(\pi^{\prime}\right)^{-1}$ is a $p$-th power. Claim 2 follows from this and Claim 3 and from Lemma 2.4 (5) applied to the extension $L^{\prime \prime} / M^{\prime \prime}$.

\section{Some extensions of complete discrete valuation fields}

Theorem 3.1. Let $K$ be a complete discrete valuation field whose residue field $F$ is of characteristic $p>0$. Let $L / K$ be a finite Galois extension. Then there is an extension $K^{\prime} / K$ of complete discrete valuation fields satisfying the following conditions (i)-(iii). Let $F^{\prime}$ be the residue field of $K^{\prime}$.

(i) $e\left(L K^{\prime} / K^{\prime}\right)=1$.

(ii) $\left[F^{\prime}:\left(F^{\prime}\right)^{p}\right]=p$.

(iii) The map $\Omega_{F}^{1}(\log ) \rightarrow \Omega_{F^{\prime}}^{1}(\log )$ is injective (here and in the following $\Omega_{F}^{1}(\log )=F \otimes_{\mathcal{O}_{K}} \Omega_{\mathcal{O}_{K}}^{1}(\log )$ ). 
If $F$ is finitely generated over a perfect subfield $k$, we can replace (ii) by the following stronger condition (ii)'.

(ii)' There is a perfect subfield $k^{\prime}$ of $F^{\prime}$ such that $F^{\prime}$ is finitely generated and of transcendence degree 1 over $k^{\prime}$.

We will deduce Theorem 3.1 from Theorem 2.1 and the following Propositions 3.2 and 3.3.

Proposition 3.2. Let $K$ be a complete discrete valuation field whose residue field $F$ is of characteristic $p>0$. Let $\pi$ be a prime element of $K$, let $\mathcal{O}_{K^{\prime}}$ be the completion of the discrete valuation ring which is the local ring of $\mathcal{O}_{K}\left[T, U^{ \pm 1}\right] /\left(U T^{p}-\pi\right)$ at the prime ideal generated by $T$, let $K^{\prime}$ be the field of fractions of $\mathcal{O}_{K^{\prime}}$, and let $F^{\prime}$ be the residue field of $K^{\prime}$. Then we have:

(i) The $\operatorname{map} \Omega_{F}^{1}(\log ) \rightarrow \Omega_{F^{\prime}}^{1}(\log )$ is injective.

(ii) The image of this map is contained in $\Omega_{F^{\prime}}^{1}$.

Proof. Straightforward.

Proposition 3.3. Let $K$ be a complete discrete valuation field whose residue field $F$ is of characteristic $p>0$. Then there is an extension $K \rightarrow K^{\prime}$ of complete discrete valuation fields satisfying the following conditions (i)-(iii). Let $F^{\prime}$ be the residue field of $K^{\prime}$.

(i) $e\left(K^{\prime} / K\right)=1$.

(ii) $\left[F^{\prime}:\left(F^{\prime}\right)^{p}\right]=p$.

(iii) The map $\Omega_{F}^{1} \rightarrow \Omega_{F}^{1}$, is injective.

If $F$ is finitely generated over a perfect field $k$, we can replace (ii) by the following stronger condition (ii)'.

(ii)' There is a perfect subfield $k^{\prime}$ of $F^{\prime}$ such that $F^{\prime}$ is finitely generated and of transcendence degree 1 over $k^{\prime}$.

Proof. Let $\left(b_{i}\right)_{i \in I}$ be a lifting of a $p$-base of $F$ to $\mathcal{O}_{K}$. Let $A_{0}=\mathcal{O}_{K}\left[T_{i}, U ; i \in I\right]$ be the polynomial ring and set $S_{i}=b_{i}-U T_{i} \in A_{0}$. The residue field $F_{0}$ of $A_{0}$ at the prime ideal $\mathfrak{p}_{0}$ generated by $\mathfrak{m}_{K}$ is $F_{0}=F\left(T_{i}, U ; i \in I\right)$ and $\left(S_{i}, T_{i}, U ; i \in I\right)$ is a $p$-base. For integers $n \geq 0$, writing $T_{i}=T_{i, 0}$ and $S_{i}=S_{i, 0}$, define $A_{n+1}=A_{n}\left[T_{i, n+1}, S_{i, n+1} ; i \in I\right] /\left(T_{i, n+1}^{p}-T_{i, n}, S_{i, n+1}^{p}-S_{i, n} ; i \in I\right)$ inductively and $A=\lim _{n} A_{n}$. Then $A$ is an integral domain, the ideal $\mathfrak{p}$ of $A$ generated by $\mathfrak{m}_{K}$ is a prime ideal and the local ring $A_{\mathfrak{p}}$ is a discrete valuation ring. Hence, the residue field $F^{\prime}$ of $A$ at $\mathfrak{p}$ is the extension of $F_{0}$ obtained by adding $T_{i}^{1 / p^{n}}$ and $S_{i}^{1 / p^{n}}$ for all $i, n$. Let $\mathcal{O}_{K^{\prime}}$ be the completion of the discrete valuation ring $A_{\mathfrak{p}}$ and let $K^{\prime}$ be the field of fractions of $\mathcal{O}_{K^{\prime}}$.

Then $K^{\prime}$ satisfies the conditions (i)-(iii). For (i) and (ii), this is clear. We prove that (iii) is satisfied. The $F^{\prime}$-vector space $\Omega_{F}^{1}$, is one-dimensional with base $d U$. The $F$-vector space $\Omega_{F}^{1}$ is with base $d b_{i}(i \in I)$. In $\Omega_{F}^{1}$, we have $d b_{i}=T_{i} d U$. Since $T_{i}(i \in I)$ are linearly independent over $F$, we have the injectivity.

Assume that $F$ is finitely generated over a perfect field $k$. Then $I$ is finite and $F$ is a finite extension of $k\left(b_{i} ; i \in I\right)$. Let $k^{\prime} \subset F^{\prime}$ be the extension of the rational function field $k\left(S_{i}, T_{i} ; i \in I\right) \subset F_{0}=F\left(T_{i}, U ; i \in I\right)$ given by $k^{\prime}:=\bigcup_{n \geq 0} k\left(S_{i}^{1 / p^{n}}, T_{i}^{1 / p^{n}}\right)$. Then $k^{\prime}$ is perfect and $F^{\prime}$ is a finite extension of $k^{\prime}(U)$.

3.4. We prove Theorem 3.1.

Let $K_{1} / K$ be the extension in Proposition 3.2. By taking $K_{1}$ as $K$ in Proposition 3.3, let $K_{2} / K_{1}$ be the extension $K^{\prime} / K$ of Proposition 3.3. Let $K_{3} / K_{2}$ be the maximal unramified subextension of $L K_{2} / K_{2}$. Then the extension $K_{3} / K$ satisfies (ii) and (iii) of 3.1 and (ii) of Proposition 3.2.

By applying Theorem 2.1 to $L K_{3} / K_{3}$, we obtain a finite extension $K^{\prime} / K_{3}$ such that $e\left(L K^{\prime} / K^{\prime}\right)=1$ and the residue field of $K^{\prime}$ is a separable extension of that of $K_{3}$. The extension $K^{\prime} / K$ has the desired properties. If $F$ is finitely generated over a prime field $k$, the condition (ii)' is satisfied. 


\section{Review and complements on ramification groups}

We briefly recall the definition and basic properties of ramification groups. For more detail, we refer to $[1,2,9,10,12]$. We introduce the refined logarithmic conductor for a finite Galois extension of a henselian valuation field in (4.15), as a generalization of the refined Swan conductor of an abelian character in the case where the extension is cyclic. We recall the definition of the Swan conductor of an abelian character at the end of Section 4.3. In the case where the residue field is a function field of one variable over a perfect field and the ramification index of the extension is one, we compute explicitly the refined logarithmic conductor in Proposition 4.11 using Lemma 4.6.

4.1. Let $K$ be a henselian discrete valuation field and $F$ be the residue field of the valuation ring $\mathcal{O}_{K}$. Let $\bar{K}$ be a separable closure of $K$ and $G_{K}=\operatorname{Gal}(\bar{K} / K)$ be the absolute Galois group. The residue field $\bar{F}$ of $\bar{K}$ is an algebraic closure of $F$.

Let $L$ be a finite étale $K$-algebra and $r>0$ be a rational number. Let $\operatorname{Spec} \mathcal{O}_{L} \rightarrow Q$ be a closed immersion to a smooth scheme $Q$ over $\mathcal{O}_{K}$. Let $K^{\prime} \subset \bar{K}$ be a finite extension of $K$ of ramification index $e$ such that $e r$ is an integer. Then, we define a dilatation $Q_{\mathcal{O}_{K^{\prime}}}^{[e r]} \rightarrow Q_{\mathcal{O}_{K^{\prime}}}=Q \times_{\mathcal{O}_{K}} \mathcal{O}_{K^{\prime}}$ by blowing up the closed subscheme $\operatorname{Spec} \mathcal{O}_{L} \otimes_{\mathcal{O}_{K}} \mathcal{O}_{K^{\prime}} / \mathrm{m}_{K^{\prime}}^{e r}$ and by removing the proper transform of the closed fiber. After replacing $K^{\prime}$ by a separable extension if necessary, the normalization $Q_{\mathcal{O}_{K^{\prime}}}^{(e r)}$ of $Q_{\mathcal{O}_{K^{\prime}}}^{[e r]}$ has geometrically reduced closed fiber and the geometric closed fiber $Q_{\bar{F}}^{(r)}=Q_{\mathcal{O}^{\prime}}^{(e r)} \times_{\mathcal{O}^{\prime}}, \bar{F}$ is independent of such $K^{\prime}$.

The finite set $F^{r}(L)=\pi_{0}\left(Q_{\bar{F}}^{(r)}\right)$ of connected components is independent of $Q$. If $F(L)=\operatorname{Mor}_{K}(L, \bar{K})$, the mapping $F(L) \rightarrow F^{r}(L)$ induced by the canonical morphism $\operatorname{Spec}\left(\mathcal{O}_{L} \times_{\mathcal{O}_{K}} \mathcal{O}_{K^{\prime}}\right)^{-} \rightarrow Q_{\mathcal{O}_{K^{\prime}}}^{(e r)}$ from the normalization is also independent of the choice and is a surjection. We say that the ramification of $L$ over $K$ is bounded by $r$ if the surjection $F(L) \rightarrow F^{r}(L)$ is a bijection. The ramification group $G_{K}^{r} \subset G_{K}=\operatorname{Gal}(\bar{K} / K)$ is defined to be the unique closed normal subgroup such that the surjection $F(L) \rightarrow F^{r}(L)$ induces a bijection $F(L) / G_{K}^{r} \rightarrow F^{r}(L)$.

4.2. A logarithmic variant is defined as follows. Let $L$ be a finite separable extension of $K$. Let $m$ be an integer divisible by the ramification index $e_{L / K}$ and $\pi$ be a prime element of $K$. We define an extension $K_{m}$ to be the tamely ramified extension $K[t] /\left(t^{m}-\pi\right)$ if $m$ is invertible in $F$ and to be the fraction field of the henselization of $\mathcal{O}_{K}\left[u^{ \pm 1}, t\right] /\left(u t^{m}-\pi\right)$ at the prime ideal $(t)$. Then, the finite set $F^{m r}\left(L \otimes_{K} K_{m}\right)$ is independent of such $m$ and we define $F_{\log }^{r}(L)$ to be $F^{m r}\left(L \otimes_{K} K_{m}\right)$. We say that the $\log$ ramification of $L$ over $K$ is bounded by $r$ if the surjection $F(L) \rightarrow F_{\log }^{r}(L)$ is a bijection. The ramification group $G_{\log , K}^{r} \subset G_{K}$ is defined to be the unique closed normal subgroup such that the surjection $F(L) \rightarrow F_{\log }^{r}(L)$ induces a bijection $F(L) / G_{\log , K}^{r} \rightarrow F_{\log }^{r}(L)$

Define closed normal subgroups $G_{K}^{r+} \subset G_{K}^{r}$ and $G_{\log , K}^{r+} \subset G_{\log , K}^{r}$ to be the closures of the unions $\bigcup_{s>r} G_{K}^{s}$ and $\bigcup_{s>r} G_{\log , K}^{s}$ and set $F^{r+}(L)=F(L) / G_{K}^{r+}$ and $F_{\log }^{r+}(L)=F(L) / G_{K, \log }^{r+}$. We say that the ramification (resp. the $\log$ ramification) of $L$ over $K$ is bounded by $r+$ if the surjection $F(L) \rightarrow F^{r+}(L)\left(\right.$ resp. $\left.F(L) \rightarrow F_{\log }^{r+}(L)\right)$ is a bijection.

4.3. We call the largest rational number $r$ such that the ramification (resp. the $\log$ ramification) of $L$ over $K$ is not bounded by $r$ the conductor (resp. the logarithmic conductor) of $L$ over $K$. The conductor (resp. the logarithmic conductor) of $L$ over $K$ is the smallest rational number $r$ such that the ramification (resp. the $\log$ ramification) of $L$ over $K$ is bounded by $r+$. The conductor $c$ and the logarithmic conductor $c_{\log }$ satisfies the inequality $c_{\log } \leqq c$. For an extension $K^{\prime}$ of a henselian discrete valuation field $K$ of ramification index $e$, the conductor $c^{\prime}$ and the logarithmic conductor $c_{\log }^{\prime}$ of a composition field $L^{\prime}=L K^{\prime}$ over $K^{\prime}$ satisfy $c_{\log }^{\prime} \leqq e \cdot c_{\log }$ and $c^{\prime} \leqq e \cdot c$. If $L$ is the cyclic extension defined by an abelian character $\chi$ of $G_{K}$, the Swan conductor $\operatorname{Sw}(\chi)$ is defined as the logarithmic conductor of $L$ over $K$. 
Lemma 4.4. Assume that the ramification index $e_{L / K}$ is 1 . Then, for every rational number $r>0$, the canonical surjections $F_{\log }^{r}(L) \rightarrow F^{r}(L)$ and $F_{\log }^{r+}(L) \rightarrow F^{r+}(L)$ are bijections.

Proof. Since we may take $m=1$, the assertion follows.

4.5. Let $Q \rightarrow P$ be a quasi-finite and flat morphism of smooth schemes over $\mathcal{O}_{K}$ and let

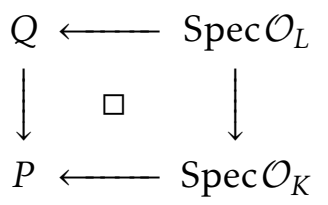

be a cartesian diagram. Then, by the functoriality of the construction of dilatations, we obtain a finite morphism $Q_{\bar{F}}^{(r)} \rightarrow P_{\bar{F}}^{(r)}$ of geometric closed fibers. Define ideals $\mathfrak{m}_{\bar{K}}^{r+} \subset \mathfrak{m}_{\bar{K}}^{r} \subset \mathcal{O}_{\bar{K}}$ by

$$
\mathfrak{m}_{\bar{K}}^{r+}=\{x \in \bar{K} \mid v(x)>r\} \subset \mathfrak{m}_{\bar{K}}^{r}=\{x \in \bar{K} \mid v(x) \geqq r\} .
$$

For a $k$-vector space $V$, let $\mathbf{V}(V)$ denote the associated covariant scheme $\operatorname{Spec} S_{k}^{\bullet} V^{\vee}$. Then, since Spec $\mathcal{O}_{K} \rightarrow P$ is a section of a smooth morphism, the conormal sheaf $N_{\mathrm{Spec}} \mathcal{O}_{K} / P$ is canonically isomorphic to the restriction of $\Omega_{P / \mathcal{O}_{K}}^{1}$ and hence the geometric closed fiber $P_{\bar{F}}^{(r)}$ is canonically identified with $\mathbf{V}\left(\operatorname{Hom}_{F}\left(\Omega_{P / \mathcal{O}_{K}}^{1} \otimes_{\mathcal{O}_{P}} F, \mathrm{~m}_{\bar{K}}^{r} / \mathrm{m}_{\bar{K}}^{r+}\right)\right)$.

The fiber $Q_{\bar{F}}^{(r)} \times_{P_{\bar{F}}^{(r)}} 0$ of the origin is canonically identified with the quotient $F^{r+}(L)=F(L) / G_{K}^{r+}$. The ramification of $L$ over $K$ is bounded by $r+$ if and only if the finite morphism $Q_{\bar{F}}^{(r)} \rightarrow P_{\bar{F}}^{(r)}$ is étale. Assume that $L$ is a Galois extension of $K$ of Galois group $G$ and fix a morphism $L \rightarrow \bar{K}$. Let $r=c$ be the conductor of $L$ over $K$. Then, the connected component $Q_{\bar{F}}^{(r) \circ}$ of $Q_{\bar{F}}^{(r)}$ containing the point corresponding to $L \rightarrow \bar{K}$ is a $G^{r}$-torsor over $P_{\bar{F}}^{(r)}$. The conductor $r=c$ of $L$ over $K$ is characterized by the condition that the morphism $Q_{\bar{F}}^{(r) \circ} \rightarrow P_{\bar{F}}^{(r)}$ is finite étale but is not an isomorphism.

Lemma 4.6. Assume that $\mathcal{O}_{L}$ is generated by one element $v \in \mathcal{O}_{L}$ over $\mathcal{O}_{K}$, set $d=\operatorname{length}_{\mathcal{O}_{L}} \Omega_{\mathcal{O}_{L} / \mathcal{O}_{K}}^{1}$ and let $\operatorname{ord}_{L}$ be the normalized valuation. Let $v^{\prime} \neq v$ be a conjugate of $v$ such that the valuation $s=\operatorname{ord}_{L}\left(v^{\prime}-v\right)$ is the largest.

1. The rational number $r=d /[L: K]+s / e_{L / K}$ equals the conductor of $L$ over $K$.

2. Let $\varphi \in \mathcal{O}_{K}[X]$ be the minimal polynomial of $v$ and define the left vertical arrow of the cartesian diagram

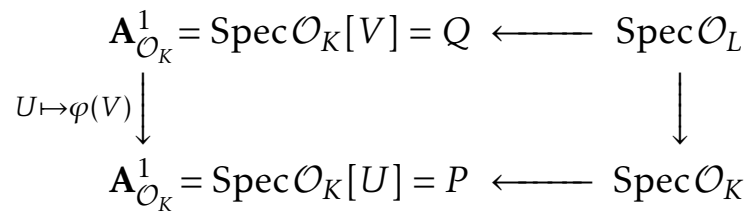

by $\varphi$ and the bottom horizontal arrow by $U \mapsto 0$. Assume that $L$ is a Galois extension of Galois group $G$ and that $G^{r}=\langle\sigma\rangle$ is cyclic of order $p$. Define isomorphisms $\mathbf{F}_{p} \rightarrow G^{r}$ by $\sigma$ and $\mathbf{A}_{\bar{F}}^{1} \rightarrow P_{\bar{F}}^{(r)}=\mathfrak{m}_{\bar{K}}^{r} / \mathrm{m}_{\bar{K}}^{r+}$ by $\varphi^{\prime}(v)(v-\sigma(v))$. Then, there is an isomorphism

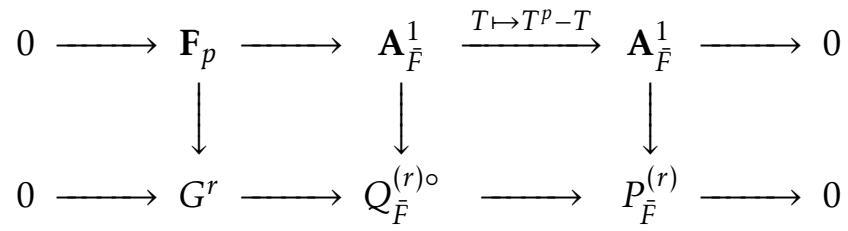

of extensions of smooth group schemes by étale group schemes. 
The proof is similar to the computation in [12, Example 3.3.3].

Proof. The left vertical arrow $Q \rightarrow P$ in (4.2) is finite flat. Let $v_{1}, \ldots, v_{n} \in L$ be the conjugates of $v$. We fix a numbering so that $v_{n}=v, v_{n-1}=\sigma(v)$ and ord ${ }_{L}\left(v_{i}-v_{n}\right)$ is increasing. Setting $X-v_{n}=\left(v_{n-1}-v_{n}\right) T$, we have $\varphi(X)=\prod_{i=1}^{n}\left(X-v_{i}\right)=\prod_{i=1}^{n}\left(v_{n}-v_{i}+\left(v_{n-1}-v_{n}\right) T\right)$. By the assumption that $G^{r}$ is cyclic of order $p$, we have $\operatorname{ord}_{L}\left(v_{i}-v_{n}\right)<\operatorname{ord}_{L}\left(v_{n-1}-v_{n}\right)$ for $i \leqq n-p$ and we may assume that $\left(v_{n-i}-v_{n}\right) /\left(v_{n-1}-v_{n}\right) \equiv i \bmod \operatorname{m}_{L}$ for $i=0, \ldots, p-1$. Hence, we have

$$
\varphi(X) \equiv \prod_{i=1}^{n-1}\left(v_{n}-v_{i}\right) \cdot \prod_{i=1}^{p-1}(1+i T) \cdot\left(v_{n-1}-v_{n}\right) T=\varphi^{\prime}(v)(v-\sigma(v))\left(T^{p}-T\right)
$$

$\bmod \mathrm{m}_{L}^{r+1}$. Thus the assertion 1 follows from the characterization of the conductor at the end of 4.5 . The assertion 2 also follows from (4.4).

Lemma 4.7. Let $P_{1}, P_{2}, Q_{1}, Q_{2}$ be smooth schemes over $\mathcal{O}_{K}$ and

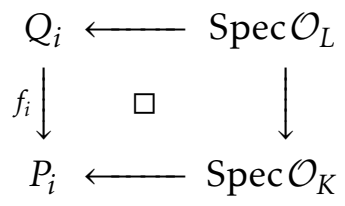

for $i=1,2$ be cartesian diagrams of schemes over $\mathcal{O}_{K}$ such that the vertical arrowes are quasi-finite and flat. Let

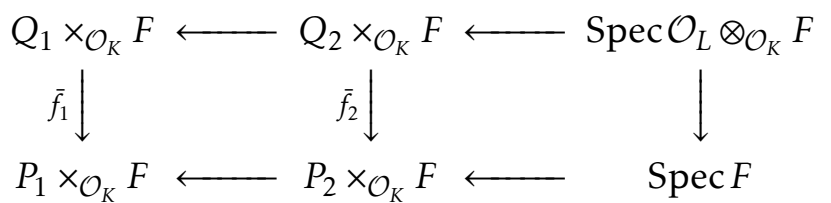

be a commutative diagram where the right square is induced by (4.5). Then for a rational number $r>0$, the diagram (4.6) induces a commutative diagram

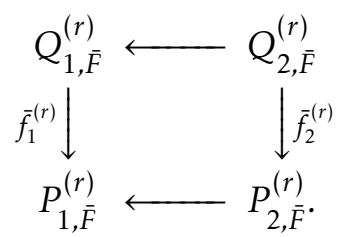

Proof. For $i=1$, 2, we consider the unions $Q_{i}^{\prime}=\left(Q_{i} \times_{\mathcal{O}_{K}} F\right) \cup \operatorname{Spec} \mathcal{O}_{L} \subset Q_{i}$ as reduced closed subschemes. Then by the commutative diagram (4.6), the morphism $Q_{1} \times_{\mathcal{O}_{K}} F \rightarrow Q_{2} \times_{\mathcal{O}_{K}} F$ and the identity of Spec $\mathcal{O}_{L}$ define a morphism $Q_{1}^{\prime} \leftarrow Q_{2}^{\prime}$. Since $Q_{1}$ is smooth over $\mathcal{O}_{K}$, after replacing $Q_{2}$ by an étale neighborhood of $\operatorname{Spec} \mathcal{O}_{L}$ if necessary, we may lift $Q_{1}^{\prime} \leftarrow Q_{2}^{\prime}$ to a morphism $Q_{1} \leftarrow Q_{2}$ over $\mathcal{O}_{K}$.

The morphism $Q_{1} \leftarrow Q_{2}$ induces a morphism of conormal modules $N_{\mathrm{Spec} \mathcal{O}_{L} / Q_{1}} \rightarrow N_{\mathrm{Spec} \mathcal{O}_{L} / Q_{2}}$ and defines a commutative diagram

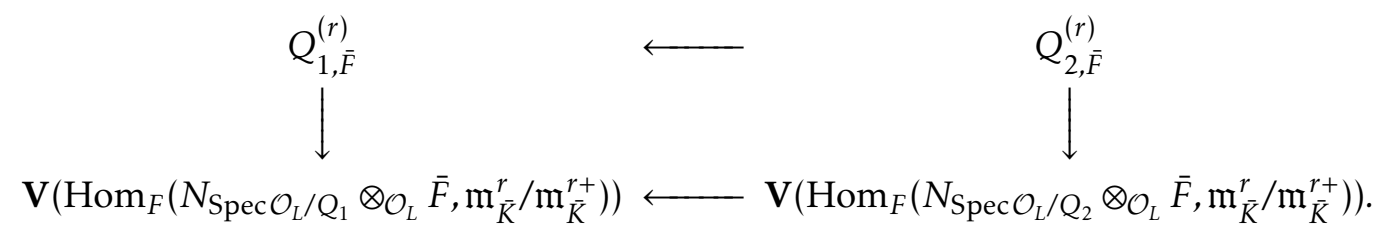

By the cartesian diagram (4.5), the conormal modules $N_{\mathrm{Spec} \mathcal{O}_{L} / Q_{i}}$ are the tensor products $N_{\mathrm{Spec} \mathcal{O}_{K} / P_{i}} \otimes_{\mathcal{O}_{K}} \mathcal{O}_{L}$ for $i=1,2$. Hence by the commutative diagram (4.6), we may replace $\mathbf{V}\left(\operatorname{Hom}_{F}\left(N_{\mathrm{Spec} \mathcal{O}_{L} / \mathrm{Q}_{i}} \otimes_{\mathcal{O}_{L}} \overline{\bar{F}}, \mathrm{~m}_{\overline{\mathrm{K}}}^{r} / \mathrm{m}_{\overline{\mathrm{K}}}^{r+}\right)\right)$ by $P_{i, \bar{F}}^{(r)}$ to get (4.7). 
By slightly enlarging the terminology, we say that the henselization of a local ring of a scheme of finite type is essentially of finite type.

Lemma 4.8 (cf. [2, Lemma 4.4, 4.5]). Let $\mathcal{O}_{K}$ be a henselian discrete valuation ring essentially of finite type and flat over $W=W(k)$ for a perfect field $k$ of characteristic $p>0$.

1. There exist a smooth scheme $P_{0}$ over $W$, a divisor $D_{0} \subset P_{0}$ smooth over $W$, a divisor $X_{0} \subset P_{0}$ flat over over $W$ meeting $D_{0}$ transversely and an isomorphism $\mathcal{O}_{X_{0}, \xi}^{h} \rightarrow \mathcal{O}_{K}$ over $W$ from the henselization of the local ring at a generic point $\xi$ of the intersection $X_{0} \cap D_{0}$.

2. Let $L$ be a finite separable extension of $K$ of ramification index e. Let $P_{0}, D_{0}, X_{0}$ be as in 1 . Further let $Q_{0}$ be a smooth scheme over $W, E_{0} \subset Q_{0}$ a smooth divisor over $W, Y_{0} \subset Q_{0}$ a divisor flat over $W$ meeting $E_{0}$ transversely and $\mathcal{O}_{Y_{0}, \eta}^{h} \rightarrow \mathcal{O}_{L}$ an isomorphism over $W$. Then, after replacing $X_{0}$ and $Y_{0}$ by étale neighborhoods of $\mathrm{Spec} \mathcal{O}_{K}$ and $\mathrm{Spec} \mathcal{O}_{L}$, there exists a cartesian diagram

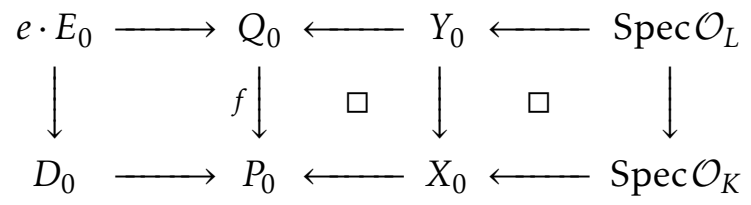

such that the vertical arrows are finite flat.

Proof. 1. Let $u_{1}, \ldots, u_{n} \in \mathcal{O}_{K}$ be liftings of a transcendental basis $\bar{u}_{1}, \ldots, \bar{u}_{n} \in F$ over $k$ such that $F$ is a finite separable extension of $k\left(\bar{u}_{1}, \ldots, \bar{u}_{n}\right)$ and $\pi$ be a prime element of $K$. If we set $\mathbf{A}_{W}^{n+1}=\operatorname{Spec} W\left[u_{1}, \ldots, u_{n}, t\right]$, the morphism $\mathcal{O}_{K} \rightarrow \mathbf{A}_{W}^{n+1}$ defined by $u_{1}, \ldots, u_{n}, \pi \in \mathcal{O}_{K}$ is formally unramified. Hence, there exists an étale neighborhood $P_{0} \rightarrow \mathbf{A}_{W}^{n+1}$ of the image $\xi$ of the closed point of $\operatorname{Spec} \mathcal{O}_{K}$, a regular divisor $X_{0} \subset P_{0}$ and an isomorphism $\mathcal{O}_{X_{0}, \xi}^{h} \rightarrow \mathcal{O}_{K}$. It suffices to define $D_{0}$ by $t$.

2. Take a function on $P_{0}$ defining $D_{0}$ and take an étale morphism $P_{0} \rightarrow \mathbf{A}_{W}^{n+1}=\operatorname{Spec} W\left[u_{1}, \ldots, u_{n}, t\right]$ such that $D_{0} \subset P_{0}$ is defined by $t$. Let $\pi \in \mathcal{O}_{K}$ be the image of $t$.

Let $s$ be a function on $Q_{0}$ defining $E_{0}$ and let $\pi^{\prime} \in \mathcal{O}_{L}$ be the image of $s$. Define $v \in \mathcal{O}_{L}^{\times}$by $\pi=v \pi^{\prime e}$ and lift it to a unit $\tilde{v}$ on $Q_{0}$. We define a morphism $Q_{0} \rightarrow \mathbf{A}_{W}^{n+1}$ satisfying $t \mapsto \tilde{v} s^{e}$ and lifting the composition $\operatorname{Spec} \mathcal{O}_{L} \rightarrow \operatorname{Spec} \mathcal{O}_{K} \rightarrow \mathbf{A}_{W}^{n+1}$. By replacing $Q_{0}$ by an étale neighborhood, we may lift $Q_{0} \rightarrow \mathbf{A}_{W}^{n+1}$ to $f: Q_{0} \rightarrow P_{0}$ satisfying $f^{*} D_{0}=e \cdot E_{0}$.

We show that the middle and the right squares are cartesian after replacing $Q_{0}$ and $P_{0}$ by étale neighborhoods. Since the residue fields $F$ and $E$ of $K$ and $L$ are the function field of the closed fibers $D_{0, k}$ and $E_{0, k}$, we may assume $E_{0} \rightarrow D_{0}$ and hence $Q_{0} \rightarrow P_{0}$ are quasi-finite and hence flat. Further, we may assume that $Q_{0} \rightarrow P_{0}$ is finite flat and the right square is cartesian. Then, the morphism $Q_{0} \rightarrow P_{0}$ is of degree $[L: K]$ and hence the middle square is cartesian.

4.9. Assume that $\mathcal{O}_{K}$ is essentially of finite type and flat over $W=W(k)$ for a perfect field $k$ of characteristic $p>0$ and let the notation be as in Lemma 4.8.2. We define a dilatation

$$
P^{\sim}=\left(P_{0} \times{ }_{W} \mathcal{O}_{K}\right)^{\sim} \rightarrow P=P_{0} \times_{W} \mathcal{O}_{K}
$$

by blowing-up $D_{0} \times_{W} F=\left(D_{0} \times{ }_{W} \mathcal{O}_{K}\right) \cap\left(P_{0} \times_{W} F\right) \subset P_{0} \times_{W} \mathcal{O}_{K}$ and by removing the proper transforms of $D_{0} \times{ }_{W} \mathcal{O}_{K}$ and of $P_{0} \times{ }_{W} F$. We consider a cartesian diagram

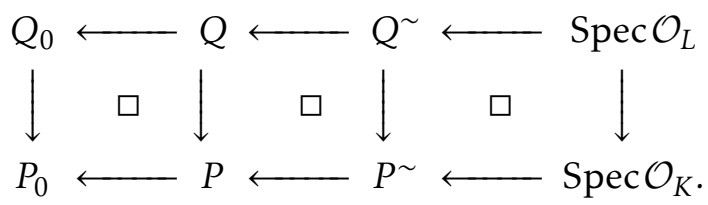

We consider $\mathcal{O}_{K}$ as a $\log$ scheme with the $\log$ structure defined by the closed point. With respect to the $\log$ structure of $Q^{\sim}$ defined by the pull-back of $E_{0}$, the $\log$ scheme $Q^{\sim}$ is $\log$ smooth over $\mathcal{O}_{K}$. Let $K^{\prime}$ be 
a finite separable extension such that the ramification index $e_{K^{\prime} / K}$ is divisible by $e=e_{L / K}$. Then, the $\log$ product $Q_{\mathcal{O}_{K^{\prime}}}^{\sim}=Q^{\sim} \times_{\mathcal{O}_{K}}^{\log } \mathcal{O}_{K^{\prime}}$ is classically smooth over $\mathcal{O}_{K^{\prime}}$ and we have a cartesian diagram

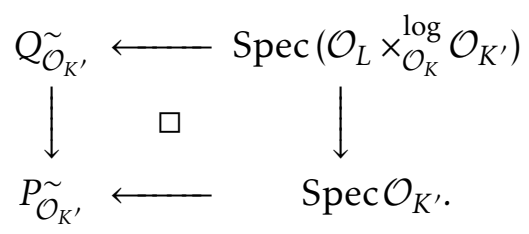

By the cartesian diagram (4.10), the conormal modules $N_{\text {Spec }} \mathcal{O}_{L} / Q$ and $N_{S p e c} \mathcal{O}_{L} / Q^{\sim}$ are the pull-backs of $N_{\mathrm{Spec} \mathcal{O}_{K} / P}=\Omega_{P_{0} / W}^{1} \otimes_{\mathcal{O}_{P_{0}}} \mathcal{O}_{K}$ and $N_{\text {Spec } \mathcal{O}_{K} / P^{\sim}}=\Omega_{P_{0} / W}^{1}\left(\log D_{0}\right) \otimes_{\mathcal{O}_{P_{0}}} \mathcal{O}_{K}$. We have an exact sequence $0 \rightarrow N_{D_{0} / P_{0}} \rightarrow \Omega_{P_{0} / W}^{1} \otimes_{\mathcal{O}_{P_{0}}} \mathcal{O}_{D_{0}} \rightarrow \Omega_{D_{0} / W}^{1} \rightarrow 0$ and a commutative diagram

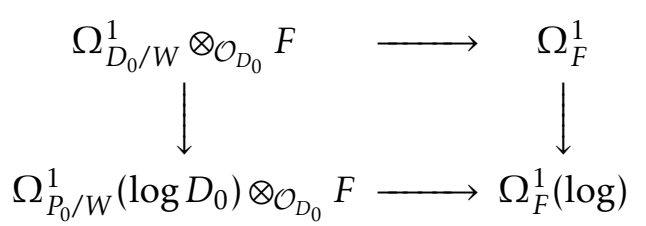

where the horizontal arrows are canonical isomorphisms. Hence the diagram (4.10) and (4.11) define a commutative diagram

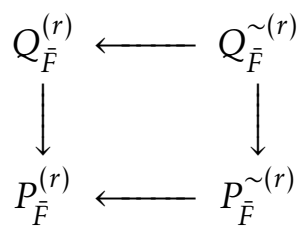

of the reduced closed fibers of dilatations. The bottom arrow is the linear mapping

$$
\begin{aligned}
& P_{\bar{F}}^{(r)}=\mathbf{V}\left(\operatorname{Hom}_{F}\left(\Omega_{P_{0} / W}^{1} \otimes_{\mathcal{O}_{D_{0}}} F, \mathfrak{m}_{\bar{K}}^{r} / \mathrm{m}_{\bar{K}}^{r+}\right)\right) \\
& \leftarrow P_{\bar{F}}^{\sim(r)}=\mathbf{V}\left(\operatorname{Hom}_{F}\left(\Omega_{F}^{1}(\log ), \mathfrak{m}_{\bar{K}}^{r} / \mathrm{m}_{\bar{K}}^{r+}\right)\right)
\end{aligned}
$$

of $\bar{F}$-vector spaces and its image is $\mathbf{V}\left(\operatorname{Hom}_{F}\left(\Omega_{F}^{1}, \mathrm{~m}_{\bar{K}}^{r} / \mathrm{m}_{\bar{K}}^{r+}\right)\right)$.

Assume that $L$ is a Galois extension of $K$ of Galois group $G$ and let $r=c_{\log }>0$ be the logarithmic conductor of $L$ over $K$. We fix a morphism $L \rightarrow \bar{K}$ over $K$. By [10, Theorem 2], the right vertical arrow of (4.12) restricted to the connected component $Q_{\bar{F}}^{\sim(r) \circ} \subset Q_{\bar{F}}^{\sim(r)}$ containing the point corresponding to $L \rightarrow \bar{K}$ defines an extension

$$
0 \longrightarrow G_{\log }^{r} \longrightarrow Q_{\bar{F}}^{\sim(r) \circ} \longrightarrow P_{\bar{F}}^{\sim(r)}=\mathbf{V}\left(\operatorname{Hom}_{F}\left(\Omega_{F}^{1}(\log ), \mathrm{m}_{\bar{K}}^{r} / \mathrm{m}_{\bar{K}}^{r+}\right)\right) \longrightarrow 0
$$

of an $\bar{F}$-vector space by an $\mathbf{F}_{p}$-vector space. By [11, Proposition 1.20], the class of the extension (4.14) defines an element

$$
\omega \in G_{\log }^{r} \otimes_{\mathbf{F}_{p}} \Omega_{F}^{1}(\log ) \otimes_{F} \mathfrak{m}_{\bar{K}}^{-r} / \mathfrak{m}_{\bar{K}}^{-r+} .
$$

This is independent of the choice of the diagram (4.10) and is called the refined logarithmic conductor of $L$ over $K$. If $G$ is cyclic and $\chi: G \rightarrow \mathbb{C}^{\times}$is an injective abelian character of $G$ and an injection $\mathbb{Z} / p \rightarrow \mathbb{C}^{\times}$is fixed, the image of $\omega$ in $\Omega_{F}^{1}(\log ) \otimes_{F} \mathfrak{m}_{\bar{K}}^{-r} / \mathfrak{m}_{\bar{K}}^{-r+}$ is the refined Swan conductor

$$
\operatorname{rsw}(\chi) \in \Omega_{F}^{1}(\log ) \otimes_{F} \mathfrak{m}_{\bar{K}}^{-r} / \mathfrak{m}_{\bar{K}}^{-r+} .
$$

Lemma 4.10. Let $L$ be a finite Galois extension of $K$ of Galois group $G$. Let $r$ be the logarithmic conductor of $L$ over $K$ and $\omega \in G_{\log }^{r} \otimes_{\mathrm{F}_{p}} \Omega_{F}^{1}(\log ) \otimes_{F} \mathrm{~m}_{\bar{K}}^{-r} / \mathfrak{m}_{\bar{K}}^{-r+}$ be the refined logarithmic conductor. 
1. If the conductor of $L$ over $K$ is the same as the logarithmic conductor $r$ of $L$ over $K$, then the refined logarithmic conductor $\omega$ is in the image of $G_{\log }^{r} \otimes_{\mathrm{F}_{p}} \Omega_{F}^{1} \otimes_{F} \mathfrak{m}_{\bar{K}}^{-r} / \mathfrak{m}_{\bar{K}}^{-r+}$.

2. Let $K^{\prime}$ be an extension of henselian valuation fields of $K$ of ramification index e and of residue field $F^{\prime}$. Assume that the image $\omega^{\prime} \in G_{\log }^{r} \otimes_{\mathbf{F}_{p}} \Omega_{F^{\prime}}^{1}(\log ) \otimes_{F^{\prime}} \mathfrak{m}_{\bar{K}^{\prime}}^{-r} / \mathfrak{m}_{\bar{K}^{\prime}}^{-r+}$ of the refined logarithmic conductor $\omega$ of $L$ over $K$ is non-trivial.

Then, the logarithmic conductor $r^{\prime}$ of a composition field $L^{\prime}$ over $K^{\prime}$ equals er and $\omega^{\prime}$ is the image of the refined logarithmic conductor of $L^{\prime}$ over $K^{\prime}$ by the morphism induced by the injection $\mathrm{Gal}\left(L^{\prime} / K^{\prime}\right)_{\log }^{r^{\prime}} \rightarrow G_{\log }^{r}$.

Proof. 1. We may assume that the residue field $F$ of $K$ is of finite type over a perfect subfield $k$. Then, the assertion follows from the commutative diagram (4.12).

2. By the functoriality of construction, the logarithmic ramification of $L^{\prime}$ over $K^{\prime}$ is bounded by er+. We may assume that the residue fields $F$ and $F^{\prime}$ of $K$ and $K^{\prime}$ are of finite type over perfect subfields $k \subset k^{\prime}$. Then, further by the functoriality of construction, we obtain a morphism $Q_{\bar{F}^{\prime}}^{\prime(e r)} \rightarrow Q_{\bar{F}}^{(r)}$ compatible with the injection $G^{\prime}=\operatorname{Gal}\left(L^{\prime} / K^{\prime}\right) \rightarrow G$ and a commutative diagram

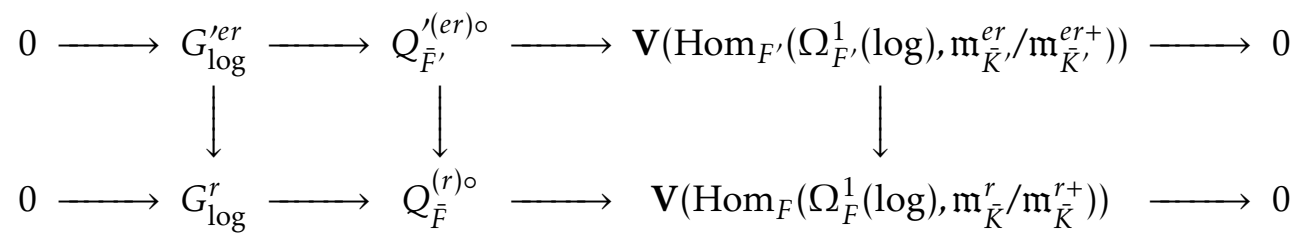

of extensions. Since $\omega^{\prime}$ is the extension class of the pull-back of the lower line by the right vertical arrow, the assumption $\omega^{\prime} \neq 0$ means that the pull-back is non-trivial and $G_{\log }^{\prime e r} \neq 0$. Hence $e r$ is the logarithmic conductor of $L^{\prime}$ over $K^{\prime}$. The last assertion also follows from the diagram (4.17).

Proposition 4.11. Assume that the residue field $F$ of $K$ is a function field of one variable over a perfect subfield $k$ of characteristic $p>0$ and that the characteristic of $K$ is 0 . Let $u \in \mathcal{O}_{K}$ be a lifting of an element $\bar{u} \in F$ such that $F$ is a finite separable extension of $k(\bar{u})$.

Let $L$ be a finite Galois extension of $K$ of Galois group $G$. Assume that the ramification index is 1 and that the residue field $E$ is a purely inseparable extension of $F$. Let $v \in \mathcal{O}_{L}$ be a lifting of a generator $\bar{v} \in E=F(\bar{v})$ and let $\varphi \in \mathcal{O}_{K}[T]$ be the minimal polynomial of $v$. Assume that $\varphi \equiv T^{q}-\bar{u} \bmod m_{K}$.

Let $r$ be the logarithmic conductor of $L$ over K. Assume that $G^{r}$ is cyclic of order $p$ and identify $G^{r}=\langle\sigma\rangle$ with $\mathbf{F}_{p}$ by fixing a generater $\sigma$. Then, $r=\operatorname{ord}_{L} \varphi^{\prime}(v)(v-\sigma(v))$ and the refined logarithmic conductor of $L$ over $K$ is

$$
\frac{d \bar{u}}{\varphi^{\prime}(v)(v-\sigma(v))} \in \Omega_{F}^{1} \otimes_{F} \mathfrak{m}_{\bar{K}}^{-r} / \mathfrak{m}_{\bar{K}}^{-r+} .
$$

Almost the same result as Proposition 4.11 is proved in [5, Theorem 5.9] in a similar way. Although we assume that $K$ is of mixed characteristic in Proposition 4.11, the same assertion is proved more easily in the equal characteristic case.

Proof. By Lemma 4.4, the logarithmic conductor equals the conductor. The equality $r=\operatorname{ord}_{L} \varphi^{\prime}(v)(v-\sigma(v))$ follows then from Lemma 4.6. We use the notation in Lemma 4.8.2. Since $D_{0} \subset P_{0}$ is smooth over $W$, there exists a smooth morphism $P_{0} \rightarrow \mathbf{A}_{W}^{1}$ such that $D_{0}$ is the pull-back of the 0 -section Spec $W \rightarrow \mathbf{A}_{W}^{1}$. By the assumption that $e=1$, the divisor $E_{0} \subset Q_{0}$ is also the pull-back of the 0 -section Spec $W \rightarrow \mathbf{A}_{W}^{1}$.

Let $P_{1}=P_{0} \times{ }_{A_{W}^{1}} \mathcal{O}_{K}$ and $Q_{1}=Q_{0} \times_{\mathbf{A}_{W}^{1}} \mathcal{O}_{K}$ be the fiber products with respect to the composition $\mathcal{O}_{K} \rightarrow P_{0} \rightarrow \mathbf{A}_{W}^{1}$. Then $P_{1}$ and $Q_{1}$ are also smooth over $\mathcal{O}_{K}$ and we have a cartesian diagram

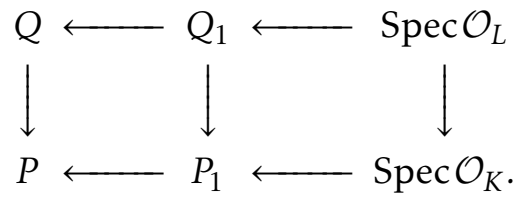


By this and Lemma 4.10, the refined logarithmic Swan conductor is the image of the class of the extension

$$
0 \rightarrow G^{r} \rightarrow Q_{1, \bar{F}}^{(r) \circ} \rightarrow P_{1, \bar{F}}^{(r)}=\mathbf{V}\left(\Omega_{F}^{1} \otimes_{F} \mathfrak{m}_{\bar{K}}^{-r} / \mathfrak{m}_{\bar{K}}^{-r+}\right) \rightarrow 0
$$

defined as the restriction of the $G^{r}$-torsor $Q_{\bar{F}}^{(r) \circ}$ over $P_{\bar{F}}^{(r)}$.

We compute the extension (4.18) by comparing it using Lemma 4.7 with that defined by the cartesian diagram (4.2) in Lemma 4.6. Since the diagram

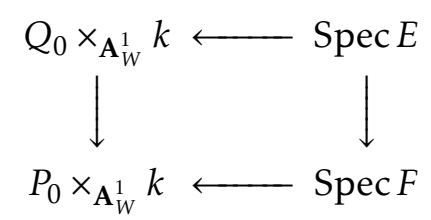

is commutative and the horizontal arrows are the canonical morphisms of the generic points, we have a commutative diagram

$$
\begin{aligned}
\operatorname{Spec} k[V]=\mathbf{A}_{k}^{1} & \longleftarrow Q_{0} \times \mathbf{A}_{W}^{1} k \\
U \mapsto V^{q} \downarrow & \downarrow \\
\text { Spec } k[U]=\mathbf{A}_{k}^{1} & \longleftarrow P_{0} \times_{\mathbf{A}_{W}^{1}} k
\end{aligned}
$$

where the horizontal arrows are étale. This induces a commutative diagram

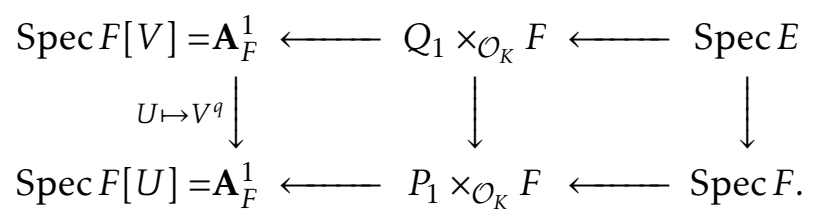

By comparing this with the cartesian diagram

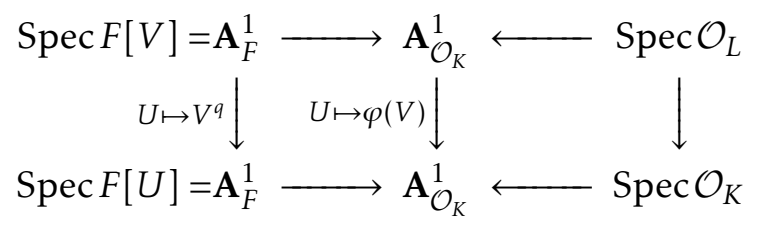

obtained by (4.2), we obtain a commutative diagram

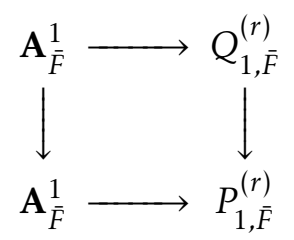

by Lemma 4.7. Since the left vertical arrow is as in Lemma 4.6 .2 and the bottom isomorphism is defined by $U-u$, the assertion follows.

\section{Coincidence of Swan conductors and of refined Swan conductors}

We prove properties of $\mathrm{Sw}^{\mathrm{ab}}$ and $\mathrm{rsw}^{\mathrm{ab}}$ similar to Lemma 4.10.2 and Proposition 4.11.

Proposition 5.1. Let $K$ be a complete discrete valuation field and let $\chi: \operatorname{Gal}(L / K) \rightarrow \mathbb{C}^{\times}$be a character for a finite abelian extension $L$ of $K$. Let $K^{\prime}$ over $K$ be an extension of complete discrete valuation fields of ramification index $e=e\left(K^{\prime} / K\right)$ and let $\chi^{\prime}: \operatorname{Gal}\left(L K^{\prime} / K^{\prime}\right) \rightarrow \mathbb{C}^{\times}$be the composition of $\chi$ with the canonical morphism $\operatorname{Gal}\left(L K^{\prime} / K^{\prime}\right) \rightarrow \operatorname{Gal}(L / K)$. 
1. We have $\mathrm{Sw}_{K^{\prime}}^{\mathrm{ab}} \chi^{\prime} \leq e \cdot \mathrm{Sw}_{K}^{\mathrm{ab}} \chi$.

2. Assume that $r=\mathrm{Sw}_{K}^{\mathrm{ab}} \chi \geq 1$. Then, the following conditions are equivalent:

(1) We have $\mathrm{Sw}_{K^{\prime}}^{\mathrm{ab}} \chi^{\prime}=e \cdot \mathrm{Sw}_{K}^{\mathrm{ab}} \chi$.

(2) The image of $\mathrm{rsw}^{\mathrm{ab}} \chi$ by the canonical morphism

$$
\mathfrak{m}_{K}^{-r} / \mathfrak{m}_{K}^{-r+1} \otimes_{\mathcal{O}_{K}} \Omega_{\mathcal{O}_{K}}^{1}(\log ) \rightarrow \mathfrak{m}_{K^{\prime}}^{-e r} / \mathfrak{m}_{K^{\prime}}^{-e r+1} \otimes_{\mathcal{O}_{K^{\prime}}} \Omega_{\mathcal{O}_{K^{\prime}}}^{1}(\log )
$$

is non-zero.

If the equivalent conditions hold, $\mathrm{rsw}^{\mathrm{ab}} \chi^{\prime}$ equals the image of $\mathrm{rsw}^{\mathrm{ab}} \chi$.

Proof. 1. Let $r=\mathrm{Sw}_{K}^{\mathrm{ab}} \chi$ and $\pi$ be a prime element of $K$. By [7, Proposition (6.3)], we have $\left\{\chi^{\prime}, 1+\pi^{r} \mathrm{~m}_{K^{\prime}}\right\}=0$ and the assertion follows.

2. The condition (2) is equivalent to that $\left\{\chi^{\prime}, 1+\pi^{r} T\right\} \neq 0$ in $\operatorname{Br}\left(L^{\prime}\right)$ where $L^{\prime}$ is the field of fractions of the henselization of $\mathcal{O}_{K^{\prime}}[T]_{\left(\pi^{\prime}\right)}$ for a prime element $\pi^{\prime}$ of $K^{\prime}$. Hence, this is equivalent to (1). Further, since the equality $\left\{\chi, 1+\pi^{r} T\right\}=\lambda_{\pi}(T \alpha, T \beta)$ is compatible with base change, $\operatorname{rsw}^{\mathrm{ab}} \chi^{\prime}$ equals the image of $\operatorname{rsw}^{\mathrm{ab}} \chi$.

Proposition 5.2. Let $K$ be a complete discrete valuation field such that the residue field $F$ is of characteristic $p>0$ and $\left[F: F^{p}\right]=p$. Let $\chi: \operatorname{Gal}(L / K) \rightarrow \mathbb{C}^{\times}$be a faithful character for a cyclic extension $L$ of $K$ of degree $q=p^{e}$ such that $e(L / K)=1$ and that the residue field $E$ of $L$ is a purely inseparable extension of $F$.

Let $v \in \mathcal{O}_{L}$ be a lifting of a generator $\bar{v} \in E=F(\bar{v})$ and let $\varphi \in \mathcal{O}_{K}[T]$ be the minimal polynomial of $v$. Let $\sigma \in \mathrm{Gal}(L / K)$ be an element of order $p$ and set $\bar{u}=\bar{v}^{q} \in F$ and $r=\operatorname{ord}_{L} \varphi^{\prime}(v)(v-\sigma(v))$.

Then, we have $\mathrm{Sw}^{\mathrm{ab}} \chi=r$ and

$$
\operatorname{rsw}^{\mathrm{ab}} \chi=\frac{d \bar{u}}{\varphi^{\prime}(v)(v-\sigma(v))} \in \mathfrak{m}_{K}^{-r} / \mathfrak{m}_{K}^{-r+1} \otimes_{F} \Omega_{F}^{1} \subset \mathfrak{m}_{K}^{-r} / \mathfrak{m}_{K}^{-r+1} \otimes_{\mathcal{O}_{K}} \Omega_{\mathcal{O}_{K}}^{1}(\log ) .
$$

Proof. The assertion follows from [7, Proposition (6.3)] and [6, Theorem (3.6)].

\subsection{We prove Theorems 1.3 and 1.5.}

Let $K$ be a complete discrete valuation field with residue field of characteristic $p>0$. We may assume that $K$ is of characteristic 0 . Let $L$ be a finite cyclic extension of $K$ and $\chi: G a l(L / K) \rightarrow \mathbb{C}^{\times}$be a faithful character. We may assume that $L$ is not tamely ramified. We may further assume that the residue field $F$ of $K$ is of finite type over a perfect subfield $k$, by a standard limit argument.

By Theorem 3.1, there exists an extension $K^{\prime}$ over $K$ of complete discrete valuation fields satisfying the conditions (i), (ii)' and (iii) in Theorem 3.1. Let $e=e\left(K^{\prime} / K\right)$ be the ramification index and $\chi^{\prime}: \operatorname{Gal}\left(L K^{\prime} / K^{\prime}\right) \rightarrow \mathbb{C}^{\times}$be the character induced by $\chi$. Then, by the condition (iii), the images of $\operatorname{rsw}(\chi)$ and $\operatorname{rsw}^{\mathrm{ab}}(\chi)$ are non-zero.

Hence by Proposition 5.1 and Lemma 4.10.2, we have $\mathrm{Sw}\left(\chi^{\prime}\right)=e \cdot \operatorname{Sw}(\chi)$ and $\operatorname{Sw}^{\mathrm{ab}}\left(\chi^{\prime}\right)=e \cdot \mathrm{Sw}^{\mathrm{ab}}(\chi)$. Further $\operatorname{rsw}\left(\chi^{\prime}\right)$ and $\operatorname{rsw}^{\mathrm{ab}}\left(\chi^{\prime}\right)$ are the images of $\operatorname{rsw}(\chi)$ and $\operatorname{rsw}^{\mathrm{ab}}(\chi)$ respectively. Thus the equality $\mathrm{Sw}(\chi)=\mathrm{Sw}^{\mathrm{ab}}(\chi)$ is equivalent to $\mathrm{Sw}\left(\chi^{\prime}\right)=\mathrm{Sw}^{\mathrm{ab}}\left(\chi^{\prime}\right)$. Further by the condition (iii) in Theorem 3.1, the equality $\operatorname{rsw}\left(\chi^{\prime}\right)=\operatorname{rsw}^{\mathrm{ab}}\left(\chi^{\prime}\right)$ is equivalent to $\operatorname{rsw}(\chi)=\operatorname{rsw}^{\mathrm{ab}}(\chi)$. Thus, we may assume that the conditions (i) and (ii)' in Theorem 3.1 are satisfied. In this case, the assertion follows from Propositions 5.2 and 4.11.

\section{References}

[1] A. Abbes and T. Saito, Ramification of local fields with imperfect residue fields, Amer. J. Math. 124 (2002), no. 5, 879-920. MR-1925338 
[2] A. Abbes and T. Saito, Ramification of local fields with imperfect residue fields. II. Doc. Math. (2003), extra vol. (Kazuya Kato's fiftieth birthday), 5-72. MR-2046594

[3] A. Abbes and T. Saito, Analyse micro-locale l-adique en caractéristique $p>0$. Le cas d'un trait. Publ. Res. Inst. Math. Sci., Kyoto Univ. 45 (2009), no. 1, 25-74. MR-2512777

[4] H. P. Epp, Eliminating wild ramification, Invent. Math. 19 (1973), 235-249. MR-0321929

[5] H. Hu, Ramification and nearby cycles for $\ell$-adic sheaves on relative curves, Tohoku Math. J. (2) 67 (2015), no. 2, 153-194. MR-3365369

[6] K. Kato, Swan conductors with differential values. In: Galois representations and arithmetic algebraic geometry (Kyoto, 1985/Tokyo, 1986), pp. 315-342, Adv. Stud. Pure Math., vol. 12, North-Holland, Amsterdam, 1987. MR-0948251

[7] K. Kato, Swan conductors for characters of degree one in the imperfect residue field case. In: Algebraic K-theory and algebraic number theory (Honolulu, HI, 1987), pp. 101-131, Contemp. Math., vol. 83, Amer. Math. Soc., Providence, RI, 1989. MR-0991978

[8] F.-V. Kuhlmann, A correction to Epp's paper “Elimination of wild ramification”, Invent. Math. 153 (2003), no. 3, 679-681. MR-2000472

[9] T. Saito, Wild ramification and the characteristic cycle of an $\ell$-adic sheaf, J. Inst. Math. Jussieu 8 (2009), no. 4, 769-829. MR-2540880

[10] T. Saito, Ramification of local fields with imperfect residue fields III, Math. Ann. 352 (2012), no. 3, 567-580. MR-2885588

[11] T. Saito, Wild ramification and the cotangent bundle, J. Algebraic Geom. 26 (2017), no. 3, 399-473. MR-3647790

[12] T. Saito, Ramification groups of coverings and valuations, Tunis. J. Math. 1 (2019), no. 3, 373-426. MR3907745

[13] J-P. Serre, Corps Locaux, deuxième édition, Publications de l'Université de Nancago, No. VIII, Hermann, Paris, 1968. MR-0354618

[14] L. Spriano, On ramification theory of monogenic extensions. In: Invitation to higher local fields (Münster, 1999), pp. 151-164, Geom. Topol. Monogr., vol. 3, Geom. Topol. Publ., Coventry, 2000. MR-1804932

[15] Y. Yatagawa, Equality of two non-logarithmic ramification filtrations of abelianized Galois groups in positive characteristics, Doc. Math. 22 (2017), 917-952. MR-3665400 